\title{
Aberrant Inferior Pancreatic Artery Arising From Replaced Middle Colic Artery
}

\section{Puneet Rana}

Jacobi Medical Center https://orcid.org/0000-0002-9166-2944

Shashi Singh ( $\nabla$ Shashi.Singh@nychhc.org)

Jacobi Medical Center

\section{Research Article}

Keywords: Celiac trunk quadrifurcation, Vascular variants, Replaced middle colic, Replaced Inferior pancreatic artery, Replaced Transverse pancreatic artery

Posted Date: October 21st, 2021

DOI: https://doi.org/10.21203/rs.3.rs-660441/v1

License: (1) This work is licensed under a Creative Commons Attribution 4.0 International License. Read Full License 


\section{Abstract}

Purpose: Anatomical variants of the celiac trunk are frequently encountered during angiography. The purpose of this case report is to describe a rare celiac trunk variant and additional aberrant pancreatic vasculature discovered during trauma workup.

Methods: We report a rare anatomic variant in which the celiac trunk gives rise to the middle colic artery. In addition, we report a replaced Inferior pancreatic artery variant that has not been previously described in literature.

Results: Emergent angiogram performed on a 19-year-old female, who was activated as a level 1 trauma, demonstrated the middle colic artery arising from the celiac trunk and providing a pancreatic artery branch supplying a portion of the pancreatic body and tail.

Conclusion: This case demonstrates rare arterial variants and emphasizes the importance of preoperative imaging prior to interventional procedures or abdominal surgery.

\section{Introduction}

The celiac trunk is the first major splanchnic artery of the abdominal aorta and supplies majority of the foregut including the pancreas. Of the three ventral branches of the abdominal aorta, the celiac trunk and superior mesenteric artery (SMA) are most frequently seen with branching variations. The normal branching pattern for the celiac trunk involves a trifurcation to give rise to the common hepatic artery (CHA), left gastric artery (LGA), and splenic artery (SA). Quadrifurcation of the celiac trunk occurs when there are four branches arising directly off the celiac axis. This is a rare occurrence with an incidence of up to $8 \%[9,14]$.

The blood supply to the pancreas is abundant and forms a dense vascular network with arterial anastomoses. The inferior pancreatic artery (IPA) also known as the transverse pancreatic artery is a branch of the dorsal pancreatic artery and supplies a portion of the pancreatic body and tail. The IPA typically originates from the dorsal pancreatic artery which originates from the splenic artery however variant origins from the SA, CHA, Celiac trunk, and SMA have been documented [1-3].

Failure to recognize the presence of these vascular anomalies during invasive procedures could have devastating consequences. Thus, it is important to meticulously review preoperative imaging prior to interventional procedures or abdominal surgeries.

\section{Case Report}

We report a case of a 19-year-old female with no known past medical history who presented as a level 1 trauma after being ejected from a high-speed motor vehicle crash. After initial trauma resuscitation and blood transfusions, CT of the whole body was performed. 
MDCT (multidetector computed tomographic) angiography of the chest, abdomen and pelvis was done using a General Electric Revolution Evo scanner. The scanning parameters were as follows: $120 \mathrm{kVp}$; Auto $\mathrm{mA} ; 0.5$ second gantry rotation time; $1.375 \mathrm{~mm}$ pitch; $2.5 \mathrm{~mm}$ slice thickness. The scanning area included the chest, abdomen and pelvis.

CT angiography of the abdomen and pelvis revealed a grade 4 right hepatic lobe laceration as per the American Association for the Surgery of Trauma (AAST) classification. Active bleeding from several branches of the right hepatic artery (RHA) was visualized. Other injuries included a splenic laceration, comminuted pelvic fractures, and active extravasation from bilateral internal iliac arteries.

Upon detailed review of the vascular anatomy, CT angiography revealed a quadrifurcation of the celiac trunk giving rise to the CHA, LGA, SA, and MCA (Fig. 1). In addition, an arterial branch arising from the replaced MCA was visualized to provide blood supply to part of the pancreatic body and tail, following the course and distribution of the IPA.

Patient underwent emergent angiography and embolization of the actively bleeding vessels. Digital subtraction angiogram (DSA) of the celiac trunk demonstrated a fourth vessel providing arterial supply to the transverse colon representing a replaced MCA (Fig. 2a). Additionally, a branch was seen arising from the the replaced MCA and traveling towards the right upper quadrant of the abdomen representing the replaced IPA seen on CT angiography. DSA of the SMA revealed an absence of the MCA (Fig. 2b).

\section{Discussion}

It is hypothesized that during embryological development, splanchnic arteries arising from the dorsal aorta can undergo unusual development secondary to retention, disappearance, or translocation of parts of the primitive arterial plexus [10-13].

Of the rare celiac trunk variants, quadrifurcation represents branching of the celiac trunk into four vessels. Macro-Clement initially described this as a Type 1c celiac trunk variant [4]. Quadrifurcations of the celiac trunk have since been further categorized as common and uncommon variants [8]. A common quadrifurcation occurs when a fourth branch is added to the celiac trunk, while an uncommon quadrifurcation is characterized as those which are missing a normal celiac branch. Quadrifurcation of the celiac trunk with the addition of the middle colic artery (MCA) is a very rare variation described in cadaveric studies with a frequency of $0.5-1 \%$ and has been termed the Bergman Type 3 variation [5-6, 14-15]. The anomalous branch can be one of the two inferior phrenics, the dorsal pancreatic artery, the gastroduodenal artery or the middle colic artery found in $1.2-4.8 \%$ [12].

Vascular supply to the pancreas is complex, thus it is important to meticulously evaluate arterial supply prior to any pancreatic intervention. Variants of the IPA with aberrant origins arising from the SA, CHA, Celiac trunk, and SMA have been seen and described [1-3,7]. However, an aberrant IPA arising from the MCA has never been documented. 
To emphasize the importance of personalized imaging based preoperative evaluation we present a case that has not been previously described in literature - in which a quadrifurcation of the celiac trunk gives rise to a replaced MCA that provides arterial supply to the pancreas via an aberrant IPA.

\section{Conclusion}

In conclusion, we report a rare quadrifurcation of the celiac trunk with an aberrant branching pattern of the IPA. No previous case report has described an IPA arising from a replaced MCA which brings the most interest in the current work. This case highlights the role of ersonalized imaging based management with CT angiography is an important tool that should be used to reduce morbidity and mortality during interventional procedures and major surgeries such as colectomies and pancreatectomies.

\section{Declarations}

Funding: Not applicable

Conflicts of Interest / Competing Interests: Not applicable

Ethics Approval: Excluded from IRB

Consent to participate: Not applicable

Consent for publication: Yes

Availability fo data and materials: Not applicable

Code availability: Not applicable

Authors contribution: All authors were equally involved in writing the manuscript and selecting the images.

\section{References}

1. Bertelli E, Di Gregorio F, Bertelli L, Civeli, L et al (1996). The arterial blood supply of the pancreas: a review. II. The posterior superior pancreaticoduodenal artery. An anatomical and radiological study. Surg Radiol Anat 18(1): 1-9. https://doi.org/10.1007/BF03207753

2. Bertelli E, Di Gregorio F, Bertelli L et al (1996). The arterial blood supply of the pancreas: a review. III. The inferior pancreaticoduodenal artery. An anatomical review and a radiological study. Surg Radiol Anat 18(2): 67-74. https://doi.org/10.1007/BF01795221

3. Bertelli E, Di Gregorio F, Mosca S et al (1998). The arterial blood supply of the pancreas: a review. V. The dorsal pancreatic artery. An anatomic review and a radiologic study. Surg Radiol Anat 20(6): 445-452. https://doi.org/10.1007/BF01653138 
4. Marco-Clement I, Martinez-Barco A, Ahumada $\mathrm{N}$ et al (2016). Anatomical variations of the celiac trunk: cadaveric and radiological study. Surg Radiol Anat 38(4): 501-510. https://doi.org/10.1007/s00276-015-1542-4

5. Maleux G, Van Geet, C (2010). Replaced middle colic artery originating from the coeliac trunk. Pediatr Radiol 40 Suppl 1: S121. https://doi.org/10.1007/s00247-010-1727-2

6. Nelson TM, Pollak R, Jonasson $O$ et al (1988), Anatomic variants of the celiac, superior mesenteric, and inferior mesenteric arteries and their clinical relevance. Clin Anat 1: 75-91. https://doi.org/10.1002/ca.980010202

7. Rong GH, Sindelar WF (1987). Aberrant peripancreatic arterial anatomy. Considerations in performing pancreatectomy for malignant neoplasms. Am Surg 53(12): 726-729.

8. Rusu MC, Jianz AM, Dincă D et al (2020). Quadrifurcation Variants of the Celiac Trunk. Ann Vasc Surg. https://doi.org/10.1016/j.avsg.2020.11.016

9. Santos P, Barbosa A, Targino VA et al (2018). ANATOMICAL VARIATIONS OF THE CELIAC TRUNK: A SYSTEMATIC REVIEW. Arq Bras Cir Dig 31(4): e1403. https://doi.org/10.1590/0102$672020180001 \mathrm{e} 1403$

10. Stimec BV, Terraz S, Fasel JH (2011). The third time is the charm-anastomosis between the celiac trunk and the left colic artery. Clin Anat 24(2): 258-261. https://doi.org/10.1002/ca.21081

11. Tandler J (1903) Zur Entwicklungsgeschichte der menschlichen Darmarterien. Anatomische Hefte 23:188-210. https://doi.org/10.1007/BF02109984

12. Vandamme JP, Bonte J (1985). The branches of the celiac trunk. Acta anatomica, 122(2), 110-114. https://doi.org/10.1159/000145991

13. Wang $Y$, Cheng C, Wang L et al (2014). Anatomical variations in the origins of the celiac axis and the superior mesenteric artery: MDCT angiographic findings and their probable embryological mechanisms. Eur Radiol 24(8): 1777-1784. https://doi.org/10.1007/s00330-014-3215-9

14. Yíldírím M, Celik HH, Yíldíz $Z$ et al (2004). The middle colic artery originating from the coeliac trunk. Folia morphologica 63(3): 363-365.

15. Yoshida T, Suzuki S, Sato T (1993). Middle mesenteric artery: an anomalous origin of a middle colic artery. Surg Radiol Anat 15(4): 361-363. https://doi.org/10.1007/BF01627894

\section{Figures}



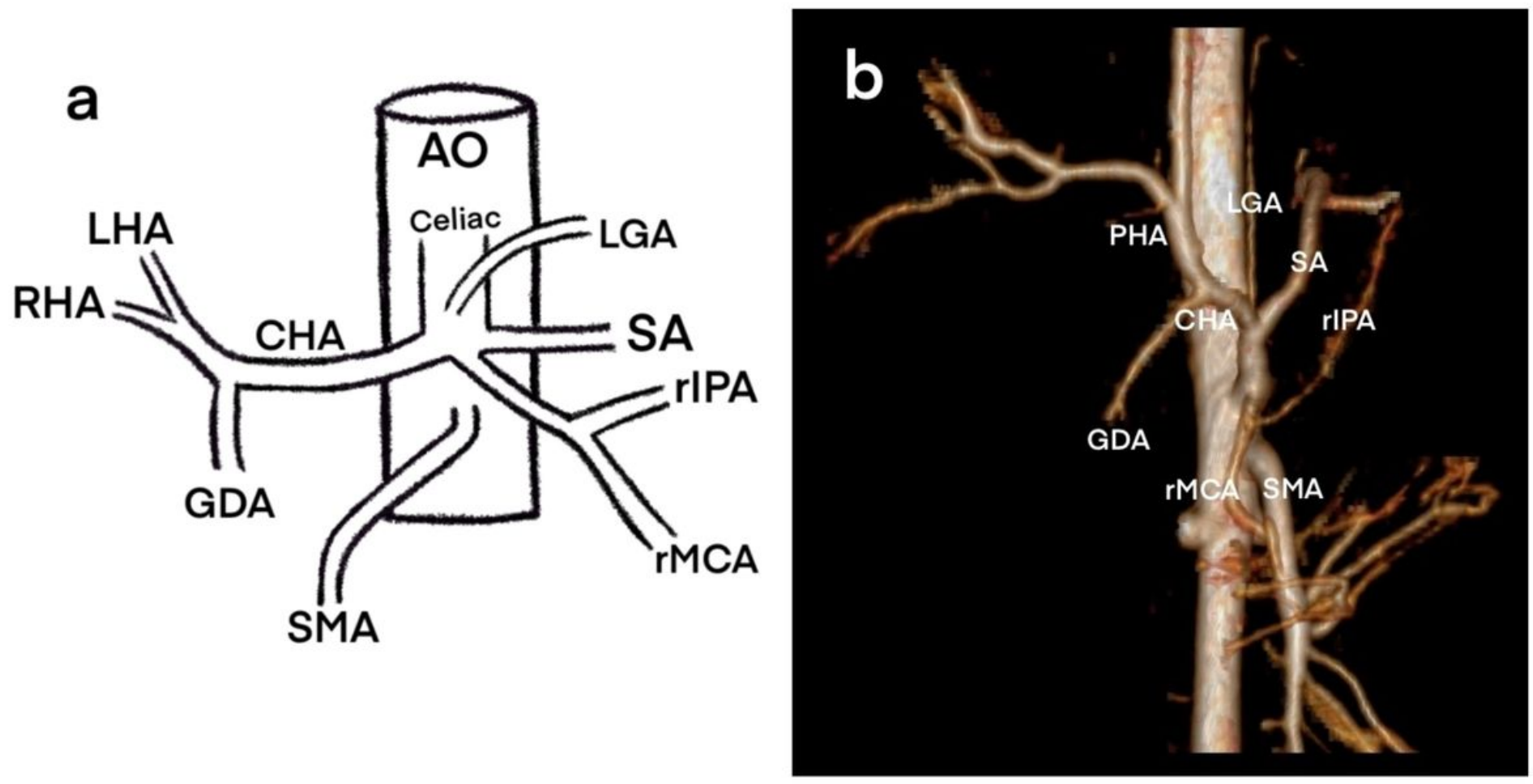

Figure 1

Quadrifurcation of the celiac trunk with the addition of the middle colic artery. Aberrant origin of the transverse pancreatic artery arising from the middle colic artery. a Schematic drawing of the abdominal aorta (AO), celiac trunk, common hepatic artery (CHA), gastroduodenal artery (GDA), left hepatic artery (LHA), right hepatic artery (RHA), left gastric artery (LGA), splenic artery (SA), replaced middle colic artery (rMCA), replaced inferior pancreatic artery (rIPA), superior mesenteric artery. b 3D reconstruction of a CT angiogram demonstrates an oblique view of the abdominal aorta. There is quadrifurcation of the celiac trunk giving rise to the splenic ar-tery (SA), left gastric artery (LGA), common hepatic artery (CHA), and replaced middle colic artery (rMCA). Replaced inferior pancreatic artery (rIPA) is seen as a branch of the rMCA. Gas-troduodenal artery (GDA), proper hepatic artery (PHA), and superior mesenteric artery (SMA) are also labeled. 

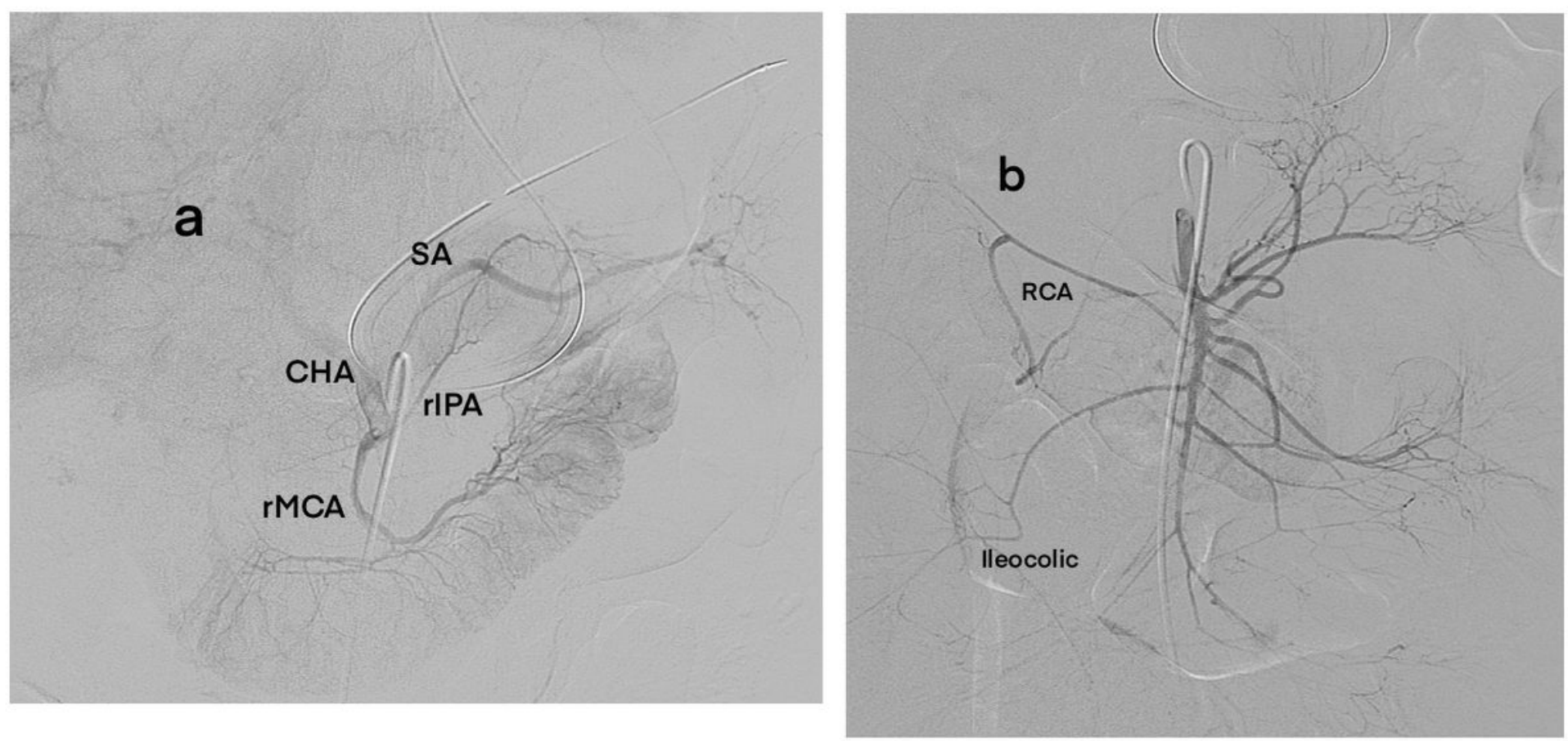

\section{Figure 2}

Anatomic anomalies of the middle colic artery and transverse pancreatic artery visualized on angiography. a Digital subtraction angiogram of the celiac trunk demonstrates a quadrifurcation providing branches to the common hepatic artery (CHA), left gastric artery (LGA), splenic artery (SA), and replaced middle colic artery ( $\mathrm{rMCA}$ ). Additionally, there is an aberrant origin of the inferior pancreatic artery (rIPA) from the MCA. $b$ Digital subtraction angiogram of the superior mesenteric artery demonstrates ileocolic and right colic arteries (RCA). There is an absence of the middle colic artery. 